\title{
Membranas de poliétersulfona/argila e sua permeabilidade à água
}

\section{Polyethersulfone/clay membranes and its water permeability}

\author{
Thamyres Cardoso de Cavalho ${ }^{1}$, Vanessa da Nobrega Medeiros ${ }^{1}$, \\ Amanda Melissa Damião Leite ${ }^{2}$, Edcleide Maria de Araújo ${ }^{1}$, \\ Hélio Lucena Lira ${ }^{1}$
}

\author{
${ }^{1}$ Unidade Acadêmica de Engenharia de Materiais, UAEMa/CCT/UFCG, Rua: Aprígio Veloso, 882, cep 58429-140, \\ Campina Grande, PB. e-mail: thamyrescc@gmail.com; vanismedeiros@gmail.com; edcleide.araujo@ufcg.edu.br; he- \\ lio.lira@ufcg.edu.br \\ ${ }^{2}$ Escola de Ciência e Tecnologia - ECT, cep: 59078-97, Natal, PB \\ e-mail: amanda.leite@ect.ufrn.br.
}

\section{RESUMO}

Membranas podem ser consideradas películas poliméricas ou inorgânicas que funcionam como uma barreira semipermeável para uma filtração em escala molecular, separando duas fases e restringindo, total ou parcialmente, o transporte de uma ou várias espécies químicas (solutos) presentes na solução. Portanto, o objetivo deste trabalho é produzir membranas de poliétersulfona (PES) e poliétersulfona / argila pela técnica de inversão de fase e avaliar a presença de argila na obtenção de membranas para o tratamento de efluentes líquidos. O solvente utilizado foi a dimetilformamida (DMF) e argilas utilizadas foram Brasgel PA (MMT) e Cloisite $\mathrm{Na}(\mathrm{CL} \mathrm{Na})$ nas proporções de 3 a 5\% (em peso). Por difração de raios-X (DRX), as membranas com 3\% das argilas MMT e CL Na aparentemente apresentaram estruturas parcialmente esfoliadas; para a composição com 5\% de CL Na observou-se um pequeno pico, que indica que esta é, possivelmente, uma estrutura intercalada ou microcompósito. A partir dos resultados de microscopia eletrônica de varredura (MEV), visualizou-se que a superfície da membrana de PES puro apresentou uma estrutura aparentemente sem poros, no aumento utilizado e, uma superfície sem rugosidade quando comparada com às membranas com argila. As medidas de ângulo de contato indicaram que a inclusão da argila alterou a capacidade de molhamento das membranas. O fluxo com água destilada para todas as membranas iniciou alto e ao longo do tempo chegou a um patamar de estabilização. Assim, pôde-se concluir que a presença e o teor de argila alteraram a morfologia da membrana, contribuindo para um aumento do fluxo de água.

Palavras-chave: Membranas, poliétersulfona, nanocompósitos, efluentes líquidos.

\section{ABSTRACT}

Membranes can be considered polymeric or inorganic films that function as a semipermeable barrier to filtration on a molecular scale, separating two phases and restricting, totally or partially, the transportation of one or more chemical species (solute) present in the solution. Therefore, the aim of this work is to produce polyethersulfone membranes (PES) and polyethersulfone/clay by phase inversion technique and evaluate the presence of clay in obtaining membranes for wastewater treatment. The used solvent was dimethylformamide (DMF) and clays were Brasgel PA (MMT) and Cloisite Na (CL Na) in the proportion of 3 to 5\% (wt.). By Xray diffraction (XRD), the membranes with $3 \%$ of MMT and CL Na clays apparently had partially exfoliated structures. For the composition with $5 \%$ of $\mathrm{CL} \mathrm{Na}$ a small peak was observed, which indicates that this is possibly an intercalated structure or microcomposite. By scanning electron microscopy (SEM), visualizes that the pure suface of the pure PES membrane a structure apparently without pores was observed in the used magnification and without roughness surface when compared to membranes with clay. The measurements of contact angle indicated that the inclusion of clay altered the wetting ability of the membranes. The flow with distilled water for all membranes started high and over time reached a stabilization level. Thus, it can be concluded that the presence and the content of clay altered the morphology of the membrane, contributing to an increase in water flow. 
Keywords: Membranes, polyethersulfone, nanocomposites, wastewater treatment.

\section{INTRODUÇÃO}

O papel de ciência e tecnologia de membranas que, atualmente, é indicado como um desenvolvimento sustentável está se tornando cada vez mais evidente e aceito em níveis políticos e industriais [1]. Nos últimos anos, membranas têm sido extensivamente usadas em processos comerciais para separação de gases, líquidos e sólidos. Um dos fatores principais de controle para que a membrana possa separar espécies é a sua morfologia [2]. Os processos de separação por membranas têm uma enorme potencialidade, em particular, tratamento de efluentes industriais. Por isso, existe um crescente interesse nas pesquisas que envolvem a síntese de membranas poliméricas. Podem-se enumerar como as motivações para o estudo da síntese de membranas a fim de serem aplicadas para o tratamento de água: a grande redução do custo de construção e operação [1], baixo consumo de energia, redução do número de etapas em um processamento, a maior eficiência na separação e a alta qualidade do produto final [3], tornando a tecnologia de membranas competitiva em relação aos sistemas convencionais de tratamento [1].

Atualmente, as membranas sintéticas são principalmente fabricadas, a partir de materiais poliméricos. Os polímeros que são apropriados para preparar membranas incluem celulose, etilceluloses, poliamidas, polissulfonas, poliétersulfona, etc. [4,5]. Entre esses materiais, a poliétersulfona (PES) é um dos materiais poliméricos mais importantes e é amplamente utilizado no campo da separação [6]. O PES apresenta várias características desejadas para o preparo das membranas, tais como: boa propriedade de barreira, química e resistência mecânica [7]. A principal desvantagem da polietérsulfona é sua baixa hidrofilicidade e permeabilidade que leva ao aumento do entupimento da membrana $[8,9,10]$. Porém, recentemente, estudos têm reportado que partículas inorgânicas incorporadas na matriz de polímero podem melhorar a sua hidrofilicidade, permeabilidade, a resistência do material e assim melhorando o desempenho do polímero à base de membranas de nanocompósitos $[11,12]$.

De um modo geral, as nanopartículas inorgânicas incorporadas em polímeros orgânicos, combinam as propriedades desejáveis das nanopartículas inorgânicas (por exemplo, rigidez, e elevada resistência, condutividade eléctrica, estabilidade térmica e oxidativa) com as vantagens de polímeros orgânicos (por exemplo, capacidade de processamento, flexibilidade, tenacidade e custo). As melhorias significativas são, devido às interações interfaciais reforçada entre cadeias de polímeros e nanopartículas, que estão bem dispersos na matriz de polímerica [13,14]. As argilas mais utilizadas em nanocompósitos (polímero-argila) são aquelas que geralmente contêm minerais grupo esmectita, particularmente aquelas que possuem montmorilonita, que é uma argila hidrofílica. A sua adição a um teor muito baixo $(<10 \% \mathrm{em}$ peso) pode ser útil não só para produzir as membranas, mas também para melhorar suas propriedades, tais como o aumento da hidrofilicidade do material $[15,16,17]$.

O desenvolvimento de nanocompósitos poliméricos e os rápidos avanços no campo da nanotecnologia criaram grandes oportunidades para um importante progresso no campo da ciência e membrana. As membranas de nanocompósitos constituídos por nanomateriais inorgânicos estão se desenvolvendo, devido à sua maior durabilidade e desempenho em muitas aplicações de separação [18,19]. Membranas compostas, que contenham materiais inorgânicos de tamanho nano, misturado em uma matriz polimérica, são atraentes devido as suas propriedades melhoradas, como citadas anteriormente [20].

As membranas poliméricas têm sido amplamente aplicadas para a separação de gases devido ao seu baixo preço, boa estabilidade térmica, economia na processabilidade e alta eficiência energética $[21,22]$. No entanto, elas geralmente sofrem uma limitação entre a permeabilidade de gás e a seletividade. Uma abordagem alternativa para resolver este problema é a utilização de membranas de matriz mista que as quais podem melhorar a permeabilidade e seletividade dos gases [23].

As membranas mistas para separação de gás normalmente podem conter cargas inorgânicas em uma matriz de polímero orgânico, onde esta combinação pode oferecer uma melhoria nas propriedades físicas, térmicas e mecânicas destes materiais. As cargas inorgânicas utilizadas e apresentadas na literatura incluem zeólitas, sílicas, argilas, peneiras moleculares de carvão, carvões ativados, nanotubos de carbono, nanopartículas de óxidos metálicos, etc. [18]. Partindo deste pressuposto, foram tomados como base alguns trabalhos de pesquisadores que utilizaram membranas a partir da poliétersulfona, onde, utilizaram alguns aditivos para melhoria das propriedades de seletividade e fluxo. Alguns trabalhos já foram relatados na literatura sobre o desenvolvimento de membranas poliméricas de poliétersulfona com argila.

ARTHANAREESWARAN et al. [25] estudaram o efeito do solvente na obtenção de membranas de PES por inversão de fases. Eles avaliaram o fluxo de água pura, resistência, estabilidade mecânica e desempenho de separação destas membranas. Os pequisadores utilizaram o N,N-dimetilformamida (DMF), N- 
metil-2-pirrolidona (NMP) e dimetil sulfóxido (DMSO) como solventes e avaliaram o desempenho das membranas obtidas. A partir das análises de MEV, eles verificaram que as membranas de PES formaram uma estrutura assimétrica. O número de poros formados sobre a camada superior das membranas de PES, foi resultado do efeito combinado das propriedades termodinâmicas do sistema e da cinética de formação da membrana. A formação da camada macroporosa dessas membranas foi controlada pela taxa de difusão do solvente e do não-solvente (água pura). A ordem do fluxo de água pura da (s) membrana (s) com diferentes solventes foi a seguinte DMSO $>$ NMP $>$ DMF. A resistência da membrana e a estabilidade mecânica diminuíram com a diminuição da concentração do PES, enquanto que aumentaram com a concentração de solventes

LIANG et al. [18] produziram membranas planas a partir de PES com a incorporação de cargas inorgânicas de diferentes formas: lamelar (montmorilonita sódica (MMT)) e esféricas (nanopartículas de $\mathrm{TiO}_{2}$ ) na proporção de $2,4,10$, e $20 \%$ em peso. As membranas foram preparadas vertendo a solução polímerica em uma placa de vidro, colocado na estufa a $60^{\circ} \mathrm{C}$ por 50 min e posteriormente imersa em água e caracterizadas por TG, DSC, DRX, MEV, MET e permeabilidade. Foi observado que a inclusão da argila deslocou as curvas de TG para temperaturas maiores, o que mostra que a argila proporcionou uma maior estabilidade térmica da membrana, por aumentar a Tg. As análises por DRX mostraram que o PES é amorfo e que o pico característico da argila apareceu em todas as composições onde estava presente. Por MEV e MET, verificou-se que o aglomerado de carga inorgânica tornou-se mais evidente com o aumento da quantidade de carga. No caso de membranas de PES/MMT, as permeabilidades de $\mathrm{CO}_{2}$ e $\mathrm{CH}_{4}$ aumentaram significativamente com o aumento do teor de carga e, consequentemente, o aumento da seletividade ao gás foi muito reduzida. Em altas cargas MMT (10\% em peso), a difusão Knudsen tornou-se o mecanismo de transporte de gás predominante. Um comportamento diferente foi visto no caso do $\mathrm{PES} / \mathrm{TiO}_{2}$. A seletividade $\mathrm{CO}_{2} / \mathrm{CH}_{4}$ aumentou de 24,5 (membrana PES pura) para um valor máximo de 38,5 para a membrana com $4 \%$ de $\mathrm{TiO}_{2} \mathrm{e}$, em seguida, diminuiu com um aumento no teor de $\mathrm{TiO}_{2}$ (por exemplo, 17,3 em 20\%). A formação de vazios de interface $\mathrm{e}$ defeitos na membrana contribuíram para a elevação da permeabilidades e baixa seletividade aos gases.

MIERZWA et al. [26] avaliaram o efeito de nanopartículas de argila como aditivo (em alguns casos teve adição de $1 \%$ hexametafosfato de sódio (SHMP) na solução, sobre a morfologia e desempenho de membranas de ultrafiltração de poliétersulfona. As membranas foram preparadas com concentração de $18 \% \mathrm{em}$ peso de PES e $82 \%$ NMP, as composições com argila foram produzidas com as concentrações de $1 \%, 2,5 \%$ e $5 \%$ de peso de argila. A permeabilidade a água ultrapura foi usada para observar inicialmente o desempenho da membrana (controle), para as membranas com $1 \%$ de argila $+1 \%$ hexametafosfato de sódio (SHMP) e a membrana com $2 \%$ de argila tiveram as maiores permeabilidades. As imagens de MEV da superfície e da secção transversal da membrana foram usadas para determinar a porosidade superficial das membranas. Todas as características das membranas foram afetadas pela adição de argila e da sua concentração. Para as composições com maiores permeabilidades, observou-se que a adição da argila contribuiu para o aumento do tamanho de poro. $\mathrm{O}$ aumento da permeabilidade da membrana com $2 \%$ de argila é atribuída à sua maior superfície, o tamanho dos poros e a porosidade. As membranas com adições de argila obtiveram um maior potencial de contaminação, porém um simples procedimento de limpeza com detergente é realizado para resolver este problema

Apesar da potencialidade das membranas obtidas a partir de nanocompósitos serem utilizadas no campo da nanofiltração, poucos estudos têm sido dedicados a nesta área. Portanto, neste trabalho membranas de nanocompósitos à base de PES e diferentes concentrações de argila Brasgel PA (MMT) e Cloisite Na (CL $\mathrm{Na}$ ) foram preparadas por meio da técnica de inversão de fase e caracterizadas por difração de raio X (DRX), microscopia eletrônica de varredura (MEV), ângulo de contato e análise do fluxo.

\section{MATERIAIS E MÉTODOS}

\subsection{Materiais}

Foram utilizadas as argilas. A Argila Brasgel PA fornecida pela Bentonit União Nordeste (BUN), Campina Grande/PB e a argila comercialCloisite Na (Sódica) fornecida pela Bentonit União Nordeste (BUN) - São Paulo/SP. A matriz polimérica empregada foi a Poliétersulfona (PES) na forma de pó, com nome comercial de Veradel ${ }^{\circledR}$ 3000P, adquirida da Solvay. Para a preparação das membranas foi utilizado o solvente Dimetilformamida (DMF) obtida pela Neon. 


\subsection{Métodos}

\section{Preparação das Membranas}

Inicialmente, a Poliétersulfona (PES), foi seca em estufa a $60^{\circ} \mathrm{C}$ para a eliminação da umidade.

Para a preparação das membranas foi utilizado o método de inversão de fases. Este método consiste na mistura da Poliétersulfona (PES), ao solvente em quantidades previamente estudadas e estabelecidas. A mistura permaneceu sob agitação constante por 45 minutos no agitador UltraTurrax a uma velocidade de 16000 rpm. Para as composições que continham argila, a solução foi preparada adicionando primeiro a argila e o solvente, onde estes ficaram sob agitação constante por um período de 45 minutos após esse tempo, o polímero foi adicionado à solução e permaneceu por mais $45 \mathrm{~min}$ em agitação. A solução obtida foi vertida em uma placa de vidro e espalhada com um bastão, permanecendo exposta ao ar atmosférico por 1 minuto. Posteriormente, a mesma foi imersa em um banho de um não-solvente (água destilada), para que ocorresse a precipitação da solução e formação da membrana.

A argila e as membranas foram caracterizadas por Difração de Raios-X (DRX) e as membranas por Microscopia Eletrônica de Varredura (MEV), ângulo de contato e medida de fluxo com água destilada.

\section{TÉCNICAS DE CARACTERIZAÇÃO}

\section{Difração de raios $X(D R X)$}

O DRX para as argilas e membranas foram analisadas à temperatura ambiente em um equipamento Shimadzu XDR-6000, utilizando radiação $\operatorname{Cuk} \alpha(\lambda=1,5418 \AA$ ), tensão de $40 \mathrm{kV}$, corrente de $30 \mathrm{~mA}$, varredura entre $2 \theta$ de $1,5^{\circ}$ a $30^{\circ}$ e velocidade de varredura de $2 \%$ min.

\section{Microscopia eletrônica de varredura (MEV)}

As análises de microscopia eletrônica de varredura foram realizadas em um equipamento SSX 550 Superscan - Shimadzu. Onde foram avaliadas a superfície de topo das membranas. As superfícies das amostras foram revestidas com ouro ("sputtering" - Metalizador Shimadzu-IC-50, utilizando uma corrente de 4mA por um período de 3 minutos) com o objetivo de evitar o acúmulo de carga negativa.

\section{Ângulo de contato}

As medidas do ângulo de contato para determinar a hidrofilicidade das membranas foram obtidas pelo método da gota séssil, através de um instrumento portátil, modelo Phoenix-i da Suface Eletro Optics - SEO. O Phoenix 50 é posicionado diretamente em cima da superfície a ser analisada. A gota é formada manualmente com um dosador micrométrico. A imagem da gota séssil é captada pela câmera embutida no equipamento, onde é visualizada e avaliada no software.

\section{Medida de fluxo com água destilada}

Para avaliar o fluxo de permeação com água destilada água, foi utilizada uma célula de filtração, com uma área efetiva de cerca de 13,0 $\mathrm{cm}^{2}$ e um modelo de bomba com 1/5 HP de energia, acoplado a um sistema, que permite a circulação do fluido a partir do tanque de alimentação através da célula de membrana.

\section{RESULTADOS E DISCUSSÃO}

\subsection{Difração de raios-X (DRX) das argilas}

A Figura 1 apresenta os difratogramas de raios-X da argilas Bragel (MMT) e da Cloisite Na. Observou-se a existência de picos por volta de $20,04^{\circ}$ característicos da argila que tem a esmectita (E) como argilomineral predominante e outros picos de um mineral não esmectitico por volta de $21^{\circ}$ e $26,05^{\circ}$. Os mesmos podem ser atribuídos a presença de impurezas de quartzo (Q) nas amostras da argilas Brasgel PA (MMT). Este resultado também foi observado por Carvalho [27].

Para ângulos menores que $10^{\circ}$, observa-se o pico que representa a distância basal d001, determinado através da Lei de Bragg. Para a argila sem tratamento (MMT), o valor encontrado foi de 14,50 A. Para a argila comercial Cloisite $\mathrm{Na}$, o valor da distância basal é aproximadamente $14,82 \AA$, bem próximo do valor encontrado para a argila MMT. 


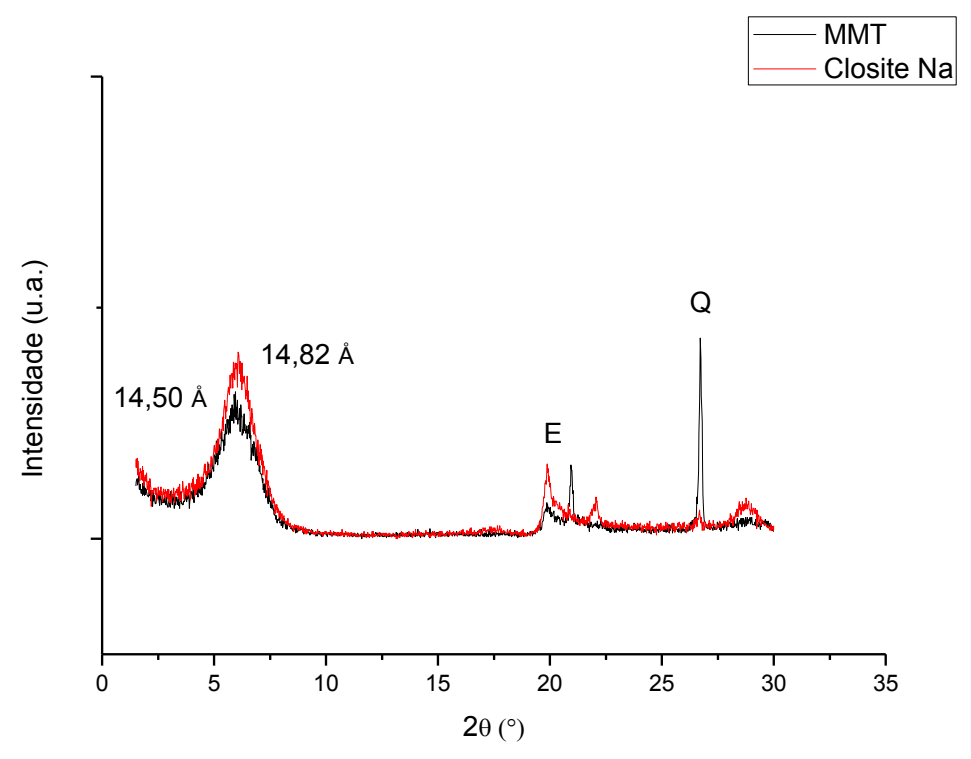

Figura 1: Difratogramas das argilas Brargel (MMT) e Cloisite Na.

\subsection{Difração de raios-X (DRX) das membranas}

A Figura 2 apresenta os difratogramas obtidos por DRX das membranas preparadas. Nota-se que a curva da membrana do polímero puro não apresenta padrão cristalino, já que o mesmo é considerado amorfo. Também é possível observar a presença de um pico de baixa intensidade, na faixa entre $15-20^{\circ}$, característico da pequena porção de fase cristalizável da matriz polimérica, como também observado por Liang et al. [18]. Para as membranas com composições contendo 3 e 5\% (em peso) de argila MMT e para a Cloisite Na 3\% pode-se observar o desaparecimento do pico característico da argila, indicando uma possível esfoliação destas membranas. Já para as membranas com 5\% de argila Cloisite $\mathrm{Na}$, observa-se a presença de um pico de baixa intensidade com distância basal de aproximadamente 15,42 , respectivamente, indicando possivelmente que esta estrutura é parcialmente intercalada.

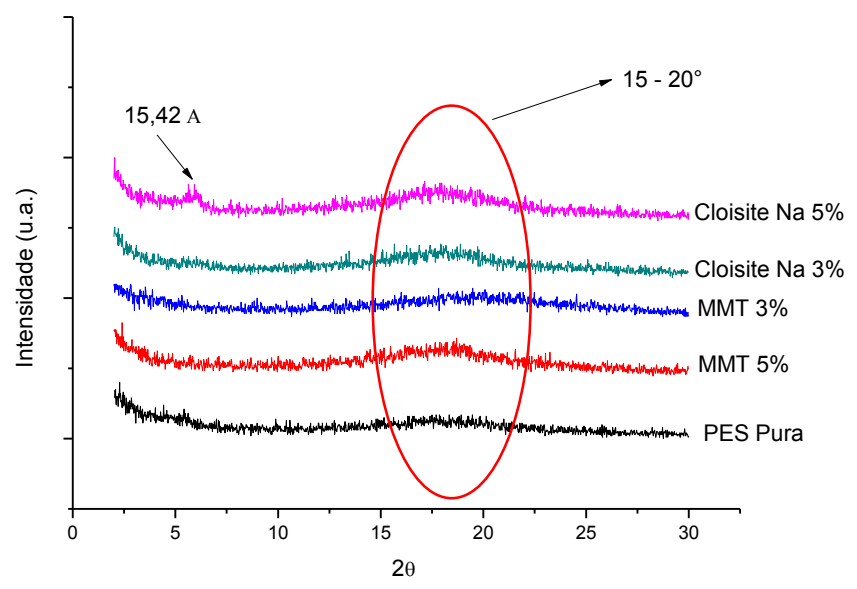

Figura 2: Difratogramas de DRX (a) das membranas de PES e de seus nanocompósitos.

\subsection{Microscopia Eletrônica de Varredura (MEV)}

As imagens de MEV da superfície das membranas de PES puro e nanocompósitos com argila MMT e Cloisite $\mathrm{Na}$, nas proporções de 3 e 5\% (em peso) são apresentados na Figura 3.

A fotomicrografia da superfície da membrana de PES puro apresentou uma estrutura aparentemente sem poros na magnificação observada e superfície sem rugosidade quando comparada às membranas com 
adição de argila. Também como observado por Wang et al. [28], todas as composições mostraram partículas na superfície, que pode ser atribuído possivelmente a uma dissolução incompleta do polímero e/ou partículas de argila, podendo ainda ser visualizado a presença de aglomerados de partículas na superfície das membranas. De acordo com Liang et al. [18], a presença destas partículas pode ser atribuída à má adesão entre PES e a carga inorgânica, devido ao caráter hidrofóbico e hidrofílico dos componentes.

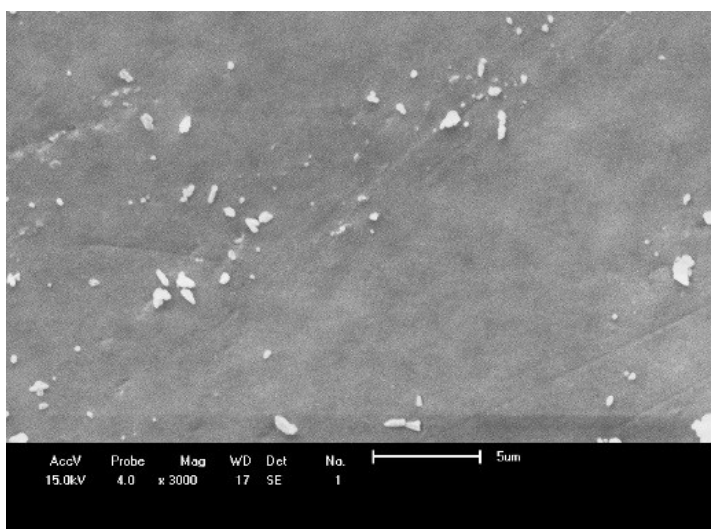

a) PES Pura

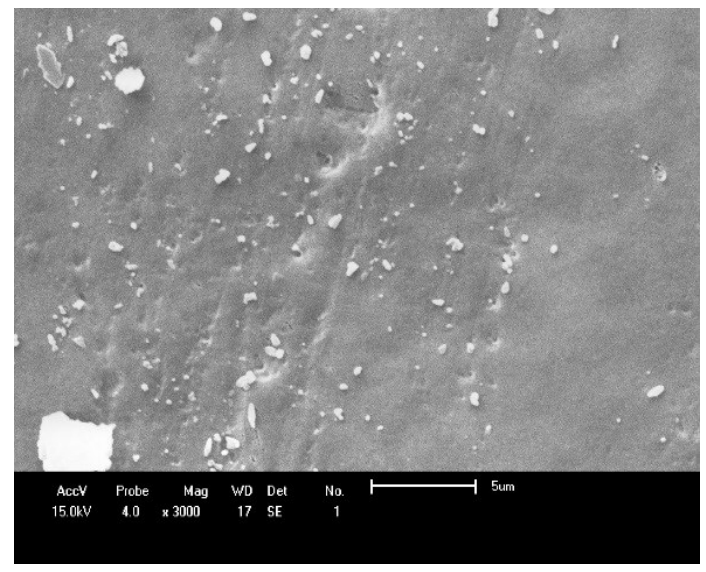

a) $\mathrm{PES} / \mathrm{MMT} 3 \%$

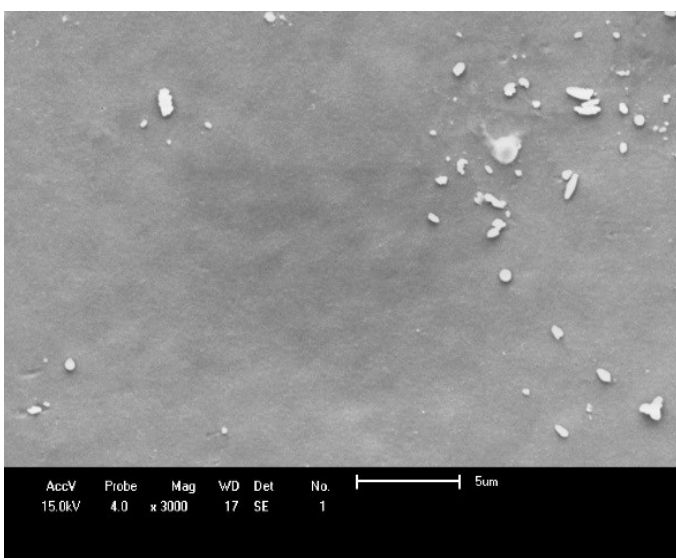

c) PES/Cloisite Na $3 \%$

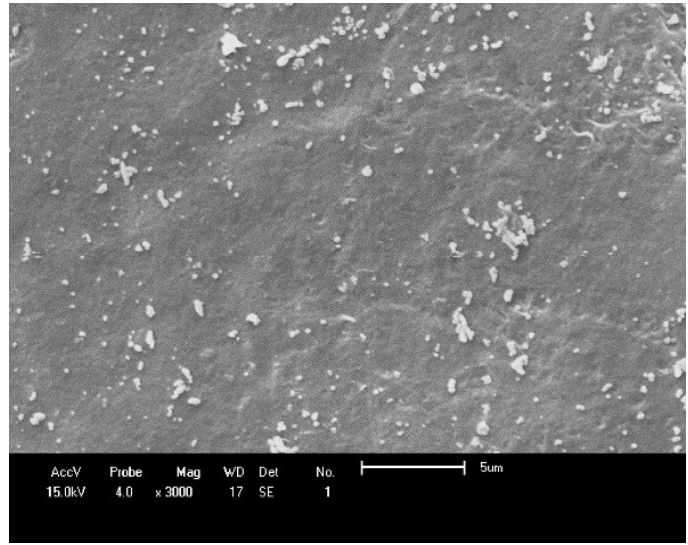

b) $\mathrm{PES} / \mathrm{MMT} 5 \%$

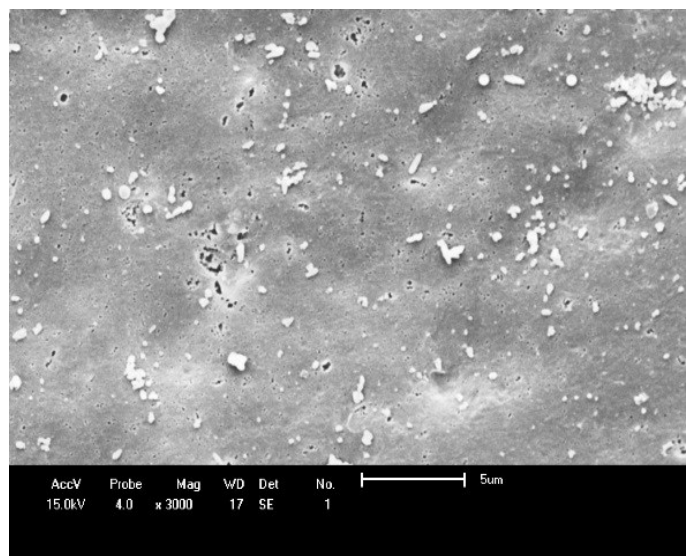

d) PES/Cloisite Na 5\%

Figura 3: Fotomicrografias de MEV da superfície das membranas de PES puro e de seus nanocompósitos.

\section{4 Ângulos de contato}

Ângulo de contato é o método mais comumente utilizado para avaliar a molhabilidade da superfície, o que depende da composição química de superfície, da rugosidade e da porosidade [29]. Na Tabela 1 estão apre- 
sentados os resultados dos ângulos de contato de uma gota de água destilada sobre as superfícies das membranas de poliétersulfona (PES), poliétersulfona com 3 e 5\% de argila Brargel (MMT) e Cloisite Na. Observou-se que o ângulo de contato com água sobre a superfície da membrana diminui com a adição de ambas as argilas. A inclusão de argila afeta diretamente a morfologia das membranas, aumentando o caráter hidrófilo delas, contribuindo assim para uma melhoria da permeação da água através da membrana.

Tabela 1: Ângulo de contato para água destilada e pata óleo das membranas de poliétersulfona.

\begin{tabular}{l|l}
\hline AMOSTRAS & $\begin{array}{l}\text { ÂNGULO DE CONTATO PARA } \\
\text { ÀGUA DESTILADA }\end{array}$ \\
\hline PES Pura & $65,26^{\circ}$ \\
\hline PES/MMT 3\% & $62,72^{\circ}$ \\
\hline PES/MMT 5\% & $62,54^{\circ}$ \\
\hline PES/Cloisite Na 3\% & $61,85^{\circ}$ \\
\hline PES/Cloisite Na 5\% & $61,81^{\circ}$ \\
\hline
\end{tabular}

\subsection{Medida de fluxo com água destilada}

O fluxo em função do tempo são visualizadas na Figura 4 e 5 para as membranas PES pura e o seus nanocompósitos, consecutivamente. Observou-se que todas as composições possuem a mesma tendência de fluxo, iniciando elevado e vai se estabilizando ao longo do tempo. A estabilidade destes fluxos ocorreu, provavelmente devido a uma compactação mecânica promovida pelas pressões aplicadas ou a um possível inchamento ocorrido nestas membranas, pois quando as mesmas entram em contato com a água, provoca uma diminuição gradativa dos poros, diminuindo assim a sua permeabilidade. Quando ocorre um decréscimo de fluxo da água destilada com o tempo, este se deve ao adensamento da microestrutura da membrana, o qual é função do valor das pressões que foram submetidas e das características estruturais destas membranas. Neste caso, foi utilizada a poliétersulfona que é um material hidrofílico, facilitando a absorção de água, reduzindo o tamanho dos poros existente na superfície destas membranas, além de contribuir diretamente com a redução do fluxo do permeado obtido [30].

Em todas as membranas, a pressão ideal à qual obteve-se estabilização com maior fluxo foi a de 1 bar. Para a pressão de 1,5 bar os fluxos foram menores, provavelmente por esta ser relativamente alta e se ter a compactação dos poros, reduzindo assim o fluxo. Observou-se que quanto maior a pressão, maior é a compactação e menor o fluxo obtido.

Analisando os fluxos estabilizados das membranas de poliétersulfona/argila, ainda pôde-se verificar que a presença da argila na matriz polimérica, influenciou no aumento destes fluxos, e que este aumento foi diretamente proporcional ao aumento no teor de argila utilizado. Além disso, as membranas híbridas com argila MMT 5\% apresentaram maiores fluxos se comparados com as demais membranas com argila, possivelmente devido à MMT possuir um maior caráter hidrofílico, por se tratar de um material com maiores índices de impurezas. Portanto, essas membranas apresentam potencial para serem aplicadas em tratamento de efluentes líquidos.

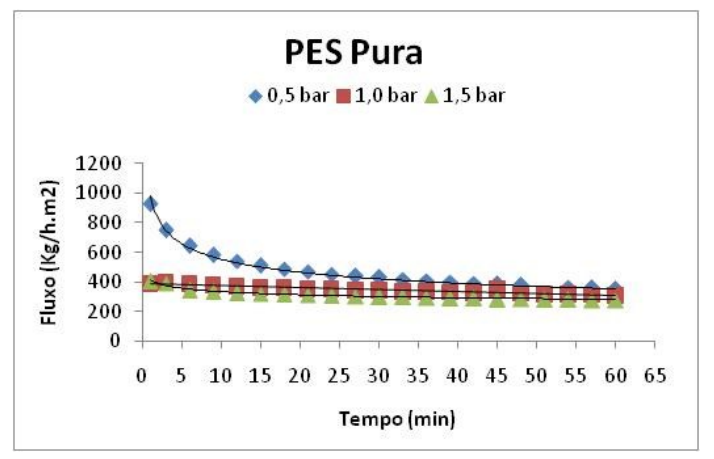

Figura 4: Gráfico do Fluxo de água destilada para as membranas de PES pura nas pressões de 0,5; 1,0 e 1,5 bar. 


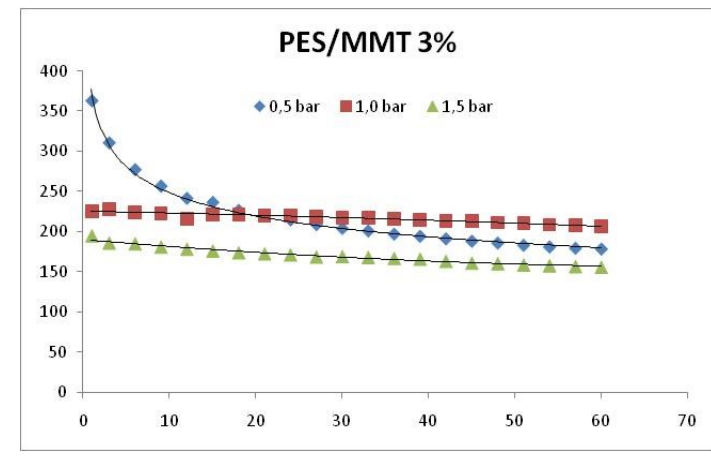

a)

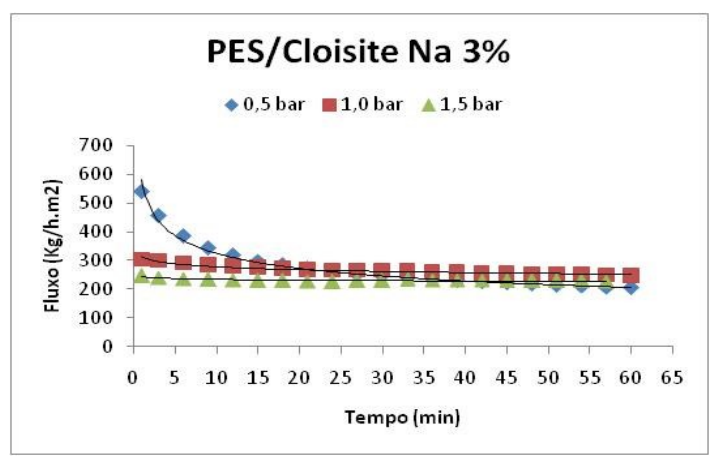

c)

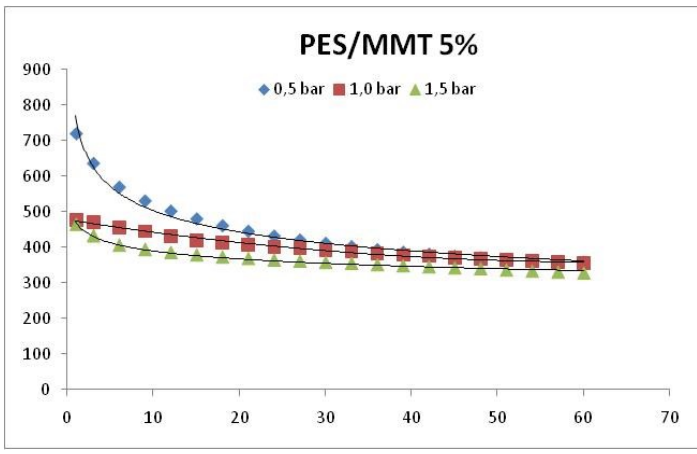

b)

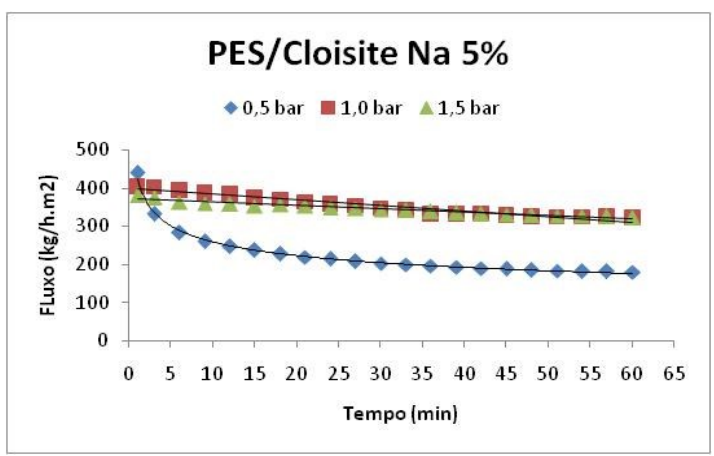

d)

Figura 5: Gráfico do Fluxo de água destilada para as membranas de PES/ argila nas pressões de 0,5; 1,0 e 1,5 bar. a) PES/MMT 3\%; b) PES/MMT 5\%; c) PES/Cloisite Na 3\%; d) PES/Cloisite Na 5\%.

\section{CONCLUSÕES}

Membranas de PES e seus nanocompósitos foram obtidas com sucesso. A partir da análise DRX, observou-se que as membranas provavelmente apresentaram nanocompósitos esfoliada e / ou parcialmente esfoliada estruturas. A partir de MEV, foi possível concluir que as membranas apresentaram uma superfície aparentemente sem poros. Todas as composições mostraram a mesma tendência do fluxo de permeado com água destilada e apresentaram maior fluxo na pressão com 1 bar. A incorporação das argilas modificou a hidrofilicidade das membranas, aumentando o fluxo permeado com água destilada. Com isso, essas membranas apresentam um grau de eficiência bastante relevante, podendo ser consideradas uma alternativa viável de tratar efluentes, capazes de separar a água de elementos indesejados no processo de microfiltração.

\section{AGRADECIMENTOS}

Os autores agradecem Bentonit União Nordeste(BUN), o DEMa/UFCG, MCTI/CNPq, PNPD/CAPES, PRH25/ANP, Petrobrás, pelo apoio financeiro.

\section{BIBLIOGRAFIA}

[1] ANADÃO, P., RABELLO, P.P., DÍAZ, V.F.R., et al., "Síntese e caracterização de membranas de microfiltração para aplicações em sistemas de tratamento de águas de abastecimento e efluentes", AIDIS de Ingeniería y Ciencias Ambientales, v. 1, n. 3, pp. 1-12, Nov. 2007.

[2] YANG, Y., ZHANG, H., WANG, P., et al., "The influence of nano-sized TiO2 fillers on the morphologies and properties of PSF UF membrane", Journal of Membrane Science, v. 288, n. 1, pp. 231-238, Fev. 2007.

[3] HABERT, A. C., BORGES, C. P., NOBREGA, R., Introdução aos Processos de Separação por Membranas, Curso Ministrado na Escola Piloto em Engenharia Química, Rio de Janeiro, COPPE/UFRJ, 1997.

[4] ANGELO, B. Handbook of membrane reactors: Fundamental materials science, design and optimization, 1 ed., UK, Wood head Publishing Limited, 2013. 
[5] ANGELO, B., ALFREDO, C., NAVIN, K.R., Advances in membrane technologies for water treatment: materials, processes and applications, UK, Wood head Publishing, Elsevier, 2015.

[6] BARTH, C., GONCALVES, M.C., PIRES, A.T.N., et al., "Symmetric polysulfone and polyethersulfone membranes: effects of thermodynamic conditions during formation on their performance", Journal of Membrane Science, v. 169, n. 1, pp. 287-99, Maio. 2000.

[7] SUSANTO, H., ULBTICHT, M., "Characteristics, performance and stability of polyethersulfone ultrafiltration membranes prepared by phase separation method using different macromolecular additives", Journalof Membrane Science, v. 327, n.1, pp.125-135, Feb. 2009.

[8] SARANYA, R., ARTHANAREESWARAN, G., DIONYSIOU, D., "Treatment of paper mill effluentusing Polyethersulfone/functionalized multi walled carbonnano tubes based nanocomposite membranes", Chemical Engineering Journal, v. 236, n. 1, pp. 369-377, Jan. 2014.

[9] BRUGGEN, V.B., "Chemical modification of polyethersulfone nanofiltration membranes: A review", Journal of Applied Polymer Science, v. 114, n. 1, pp. 630-42, June. 2009.

[10] KHULBE, K.C, FENG, C., MATSUURA, T. "The art of surface modification of synthetic polymeric membranes", Journal of Applied Polymer Science, v. 115, n. 2, pp. 855-95, 2010.

[11] WANG, Y., ZHU, J., DONG, G., et al., "Sulfonatedhalloysitenanotubes/polyethersulfonenanocompositemembrane for efficient dye purification", Separation and Purification Technology, v. 150, n.1, pp .243-251, Ago. 2015.

[12] KIM, J., BRUGGEN, B.V., "The use of nanoparticles in polymeric and ceramic membrane structures, review of manufacturing procedures and performance improvement for water treatment", Environmental Pollution, v. 158, n. 7, pp. 2335-2349, Jul. 2010.

[13] RAY, S.S., OKAMOTO., M., "Polymer/layered silicate nanocomposites: a review from preparation processing", Progress in Polymer Science, v.28, n.11, pp. 1539-1641, Ago. 2003.

[14] OKAMOTO, M., "Recent advances in polymer/layered silicate nanocomposites: an overview from science to technology", Materials Science and Technology, v. 22, n. 7, pp.756-779, Nov 2006.

[15] SCHAFER, A.I., FANE, A.G., WAITE, T. D., Nanofiltration: Principles and Applications, 1 ed., UK, Elsevier Advanced Technology, 2004.

[16] ANADAO, P., SATO, L.F., WIEBECK, H., et al., "Montmorillonite asa component of polysufone nanocomposite membranes", Applied Clay Science, v. 48, n. 1, pp.127--132, Março. 2010.

[17] MONTICELLI, O., BOTTINO, A., SCANDALE, I., et al., "Preparation and properties of polysulfone clay composite membranes", Journal of Applied Polymer Science, v. 103, n. 6, pp. 363-3644, Dez. 2007.

[18] LIANG, C.Y., UCHYTIL, P., PETRYCHKOVYCH, R., et al., "A comparison on gas separation between PES (polyethersulfone)/MMT (Na-montmorillonite) and PES/TiO2 mixed matrix membranes", Separation and Purification Technology, v.92, n.1, pp.57-63, Maio. 2012.

[19] RAHIMPOUR, A., MADAENI, S.S., TAHERI, A.H., et al., "Coupling TiO2 nanoparticles with UV irradiation for modification of polyethersulfone ultrafiltration membranes", Journal of Membrane Science. v. 313, n. 1, pp. 158-169, Abril. 2008.

[20] KIM, S., CHEN, L., JOHNSON, J.K., et al., "Polysulfone and functionalized carbono nanotube mixed matrix membranes for gas separation: theory and experiment”, Journal of Membrane Science, v. 294, n. 1, pp. 147-158, Maio. 2007.

[21] WANG, X.L., QIAN, H.J., CHEN, L.J., et al., "Dissipative particle dynamics simulation on the polymer membrane formation by immersion precipitation", Journal of Membrane Science, v. 311, n.1, pp. 251- 258, Março. 2008.

[22] KHAN, A.L., LI, X., VANKELECOM, I.F.J., "Mixed-gas CO2/CH4 and CO2/N2 separation with sulfonated PEEK membranes", Journal of Membrane Science, v. 372, n.1, pp.87-96, Abril. 2011.

[23] CHUNG, T.-S., JIANG, L.Y., LI, Y., et al., "Mixed matrix membranes (MMMs) comprising organic polymers with dispersed inorganic fillers for gas separathion", Progress in Polymer Science, v. 32, pp. 483507, Fev. 2007.

[24] ROBESON, L.M. "The upper bound revisited”, Journal of Membrane Science, v. 320, n. 1, pp.390-400, Jun. 2008. 
[25] ARTHANAREESWARAN, G., STAROV, V.M. "Effect of solvents on performance of polyethersulfone ultrafiltration membranes: Investigation of metal ion separations", Desalination, v.267, pp. 57-63, Fev. 2011.

[26] MIERZWA, J.C., ARIETA, V., VERLAGE, M., et al., "Effect of clay nanoparticles on the structure and performance of polyethersulfone ultrafiltration membranes", Desalination v.314, pp.147-158, Fev. 2013.

[27] CARVALHO, T.C., PAZ, R.A., ARAÚJO, E. M., "Avaliação do efeito do envelhecimento acelerado nas propriedades mecânicas de nanocompósitos de PA6/argila organofílica", Revista Eletrônica de Materiais e Processos, v. 9, n. 2, pp. 98-105, Maio. 2014.

[28] WANG, Y., ZHANG, B., YE, J., "Microstructures and toughening mechanisms of organo clay/polyethersulphone/epoxy hybrid nanocomposites", Materials Science and Engineering, v.528, n. 1, pp.7999- 8005, Set. 2011.

[29] ZHAO, Y., ZHANG, P., SUN, J., et al., "Electrolyte-responsive polyethersulfone membranes with zwitterionic polyethersulfone-based copolymers as additive", Journal of Membrane Science, v. 510, n. 1, pp.1-27, Jun. 2016.

[30] KOJUCH, L.R., MEDEIROS, K.M., ARAÚJO, E.M., et al., "Obtaining of polyamide 6.6 plane membrane application in oil-water separation", Materials Science Forum, v. 775-776, pp. 460-464, Jan. 2014. 